\title{
Candidemia in a major regional tertiary referral hospital - epidemiology, practice patterns and outcomes
}

Jocelyn Qi-Min Teo', Samuel Rocky Candra', Shannon Jing-Yi Lee', Shannon Yu-Hng Chia, ${ }^{1,6}$, Hui Leck', Ai-Ling Tan², Hui-Peng Neo ${ }^{1}$, Kenneth Wei-Liang Leow ${ }^{1}$, Yiying Cai ${ }^{1,3}$, Rachel Pui-Lai Ee ${ }^{3}$, Tze-Peng Lim, ${ }^{1,4}$ Winnie Lee ${ }^{1}$ and Andrea Lay-Hoon Kwa ${ }^{1,3,5^{*}}$

\begin{abstract}
Background: Candidemia is a common cause of nosocomial bloodstream infections, resulting in high morbidity and mortality. This study was conducted to describe the epidemiology, species distribution, antifungal susceptibility patterns and outcomes of candidemia in a large regional tertiary referral hospital.

Methods: A retrospective surveillance study of patients with candidemia was conducted at Singapore General Hospital between July 2012 and December 2015. In addition, incidence densities and species distribution of candidemia episodes were analysed from 2008 to 2015.
\end{abstract}

Results: In the period of 2012 to 2015, 261 candidemia episodes were identified. The overall incidence was 0.14/1000 inpatient-days. C. glabrata (31.4\%), C. tropicalis (29.9\%), and C. albicans (23.8\%) were most commonly isolated. The incidence of C. glabrata significantly increased from 2008 to 2015 (Coefficient 0.004, confidence interval $0-0.007, p=0.04)$. Fluconazole resistance was detected primarily in C. tropicalis (16.7\%) and C. glabrata (7.2\%). fks mutations were identified in one C. albicans and one C. tropicalis. Candidemia episodes caused by $C$. tropicalis were more commonly encountered in patients with haematological malignancies $(p=0.01)$, neutropenia $(p<0.001)$ and higher SAPS II scores $(p=0.02)$, while prior exposure to echinocandins was associated with isolation of $C$. parapsilosis $(p=0.001)$. Echinocandins $(73.3 \%)$ were most commonly prescribed as initial treatment. The median (range) time to initial treatment was 1 (0-9) days. The 30-day in-hospital mortality rate was 49.8\%. High SAPS II score (Odds ratio, OR 1.08; 95\% confidence interval, CI 1.05-1.11) and renal replacement therapy (OR 5.54; $\mathrm{Cl} 2.80-10.97)$ were independent predictors of mortality, while drain placement (OR 0.44; Cl 0.19-0.99) was protective.

Conclusions: Decreasing azole susceptibilities to C. tropicalis and the emergence of echinocandin resistance suggest that susceptibility patterns may no longer be sufficiently predicted by speciation in our institution. Candidemia is associated with poor outcomes. Strategies optimising antifungal therapy, especially in the critically-ill population, should be explored.

Keywords: Candida, Bloodstream infections, Antifungal susceptibility, fks, Mortality

\footnotetext{
* Correspondence: andrea.kwa.l.h@sgh.com.sg

'Department of Pharmacy, Singapore General Hospital, Blk 8 Level 2, Outram

Road, Singapore 169608, Singapore

${ }^{3}$ Department of Pharmacy, National University of Singapore, 18 Science Drive

4, Singapore 117543, Singapore

Full list of author information is available at the end of the article
} 


\section{Background}

Candida species are the leading cause of invasive fungal infections and a common cause of hospital-acquired bloodstream infections [1]. Candidemia has a profound impact on patient outcomes and the burden has increased significantly over the years. The crude mortality is high, ranging from $30-50 \%$ [2-4]; while the attributable mortality due to candidemia varied from $15-49 \%$ $[5,6]$. Increasing reports of antifungal resistance, even in newer agents such as the echinocandins, further escalate the complexity in the management of candidemia [7].

Knowledge of antifungal susceptibility patterns is imperative in the selection of early and appropriate antifungal agents for improved patient outcomes. The variable epidemiology of candidemia, contributed by the geographical and temporal variations in incidence and species distribution [4, 8-10], underscores the continuing need for local surveillance of Candida species distribution and susceptibility patterns.

Furthermore, the introduction of new echinocandins into Singapore such as anidulafungin in 2008 and micafungin in 2013, coupled with the exponential increase in echinocandin usage in our institution for the past 5 years, suggest that current susceptibility patterns should be reviewed. A recent study has also reported the emergence of echinocandin resistance in the Asia-Pacific region [11]. The objectives of this study were 1) to investigate the incidence, species distribution and antifungal susceptibilities of candidemia, and 2) to describe the clinical features and outcomes of candidemia in our population.

\section{Methods}

\section{Study setting and design}

A retrospective surveillance study of patients with candidemia was conducted at Singapore General Hospital (SGH) between July 2012 and December 2015. SGH is the largest acute care hospital (1800 beds) in the country, and covers a wide range of medical and surgical specialties. The hospital is the national/regional referral centre for services such as plastic surgery and burns, renal medicine, nuclear medicine, pathology and haematology. SGH accounts for approximately $25 \%$ of the total acute hospital beds in the public sector and $20 \%$ of acute beds nationwide.

All adult inpatients (at least 21 years old) with $\geq 1$ positive blood culture for Candida spp. were included into the study. Each positive Candida culture must be accompanied with temporally-related clinical signs and symptoms of infection for inclusion into the study. For each patient, only the first candidemia episode was recorded, unless the positive blood culture was obtained $\geq$ 30 days (with blood culture clearance and resolution of clinical features of infection of the first episode) or involved a different Candida spp. isolated from blood culture obtained $\geq 7$ days after the first episode. Episodes involving $>1$ Candida spp. isolated within 7 days of the first episode, defined as "mixed candidemia", were regarded as a single episode.

\section{Microbiology and antifungal susceptibility testing}

Candida spp. were isolated from blood using BD BACTEC $^{\mathrm{Tm}}$ FX (Becton, Dickinson and Company, Sparks, MD). The species were identified using MALDI Biotyper (BrukerDaltonik GmbH, Germany), morphology studies on cornmeal Tween 80 agar, and API 20C AUX (Biomerieux, Marcy l'Etoile, France). Isolates were stored in Microbank $^{\mathrm{TM}}$ storage vials (Pro-Lab Diagnostics, Round Rock, TX, USA) at $-70{ }^{\circ} \mathrm{C}$ until testing.

Antifungal susceptibility testing was performed using Sensititre YeastOne ${ }^{\bullet}$ YO10 panel (Trek Diagnostics System, West Sussex, England) according to manufacturer's recommendations. Minimum inhibitory concentrations (MICs) for amphotericin B, anidulafungin, caspofungin, micafungin, fluconazole, voriconazole, itraconazole, posaconazole and flucytosine were recorded. Candida krusei (Issatchenkia orientalis) ATCC 6258 and C. parapsilosis ATCC 22019 (American Type Culture Collection, Manassas, Virginia) were used as quality controls.

MICs were interpreted according to the current species-specific clinical breakpoints provided by the Clinical and Laboratory Standards Institute (CLSI) M27S4 document [12]. Where clinical breakpoints were not available, the epidemiological cut-off values (ECV) were used to classify the isolates into wild-type or non-wildtype populations [13-15].

\section{Detection of $f k s$ mutations}

Isolates classified as intermediate or resistant to echinocandins were tested for the presence of mutations in the $f k s$ genes. Hot spots 1 and 2 regions of $f k s 1$ and $f k s 2$ (for C. glabrata only) genes were amplified using polymerase chain reaction (PCR), as described previously [16].

\section{Clinical data collection}

Clinical characteristics of patients with candidemia were obtained from inpatient charts and electronic medical records using a standardised case report form. Data extracted included demographics, hospitalisation history (previous hospital stay, previous intensive care unit (ICU) stay, length of hospital stay prior to candidemia), underlying medical conditions and prior exposure to invasive interventions (central lines, urinary catheters, drainage devices, invasive ventilation, dialysis, invasive surgery, total parenteral nutrition) and medical therapy (chemotherapy, immunosuppressive therapy, antibiotics, antifungal agents) within 30 days before the first positive blood culture. Charlson comorbidity index at the time of 
admission and Simplified Acute Physiology Score (SAPS) on the day of the first positive blood culture were also recorded. Information on the management of candidemia (choice and duration of antifungal agents) and outcome (in-hospital all-cause mortality within 30 days) were collected.

\section{Data and statistical analyses}

To calculate and analyse the incidence of candidemia, the number of candidemia episodes were obtained from the clinical microbiology laboratory computerised database, while inpatient-days were obtained from the hospital administrative database. Incidence data was available from 2008 , hence trend analyses were performed for the period from 2008 to 2015 . Incidence rates were calculated as the number of candidemia episodes per 1000 inpatient-days. Linear regression was used to determine trends over time in the incidences of candidemia.

Categorical variables were presented as numbers and percentages; and were compared using the $\mathrm{X}^{2}$ or Fisher's exact test, as appropriate. Continuous variables were presented as mean \pm SD or median and range; and were compared using the Student's $t$ test, Mann-Whitney test, or Kruskal-wallis test, depending on the validity of the normality assumption.

A multivariable logistic regression model was used to identify predictors associated with 30-day mortality. Clinically plausible variables identified in the bivariate analysis were included in the multivariable logistic regression model if $p<0.1$. Significant factors which may covary were grouped and only one factor from each group was selected for entry into the model. The final model was chosen on the basis of biologic plausibility.
Odds ratios (OR) and 95\% confidence intervals (CI) were calculated to evaluate the strength of any association. For all calculations, a 2-tailed $p$ value of less than 0.05 was considered to reveal a statistical significant difference. Statistical analyses were performed using IBM SPSS Statistics for Windows, Version 23.0 (IBM Corp., Armonk, NY).

\section{Results}

\section{Incidence and species distribution}

From 2012 to 2015, 261 candidemia episodes involving 254 patients and 272 isolates were analysed. Seven patients had two separate episodes each with distinct Candida species, while a patient had a repeated episode involving the same Candida species. The incidence was 0.14 episodes per 1000 inpatient-days during the study period. C. glabrata (82/261, 31.4\%), C. tropicalis (78/261, $29.9 \%)$, C. albicans $(62 / 261,23.8 \%)$, and C. parapsilosis (36/261, 13.8\%) accounted for majority of the episodes. Other species including C. dubliniensis $(n=7), C$. krusei $(n=3)$, C. guilliermondii (Meyerozyma guilliermondii) $(n=1), C$. kefyr (Kluyveromyces marxianus) $(n=1), C$. haemulonis $(n=1)$ and C. pseudohaemulonii $(n=1)$ accounted for the remaining episodes. Of these 261 episodes, 11 (4.2\%) were mixed candidemia episodes.

The incidence density and species distribution are displayed in Fig. 1. The overall incidence density was 0.15 (range $0.12-0.18$ ) episodes $/ 1000$ inpatient-days and 0.89 (range 0.74-1.05) episodes/1000 admissions from 2008 to 2015. Analysing the incidence densities from 2008 to 2015, we found no significant change in the incidence density of candidemia [Coefficient 0.00009 , confidence interval (CI) - 0.007-0.007, $p=0.98]$.

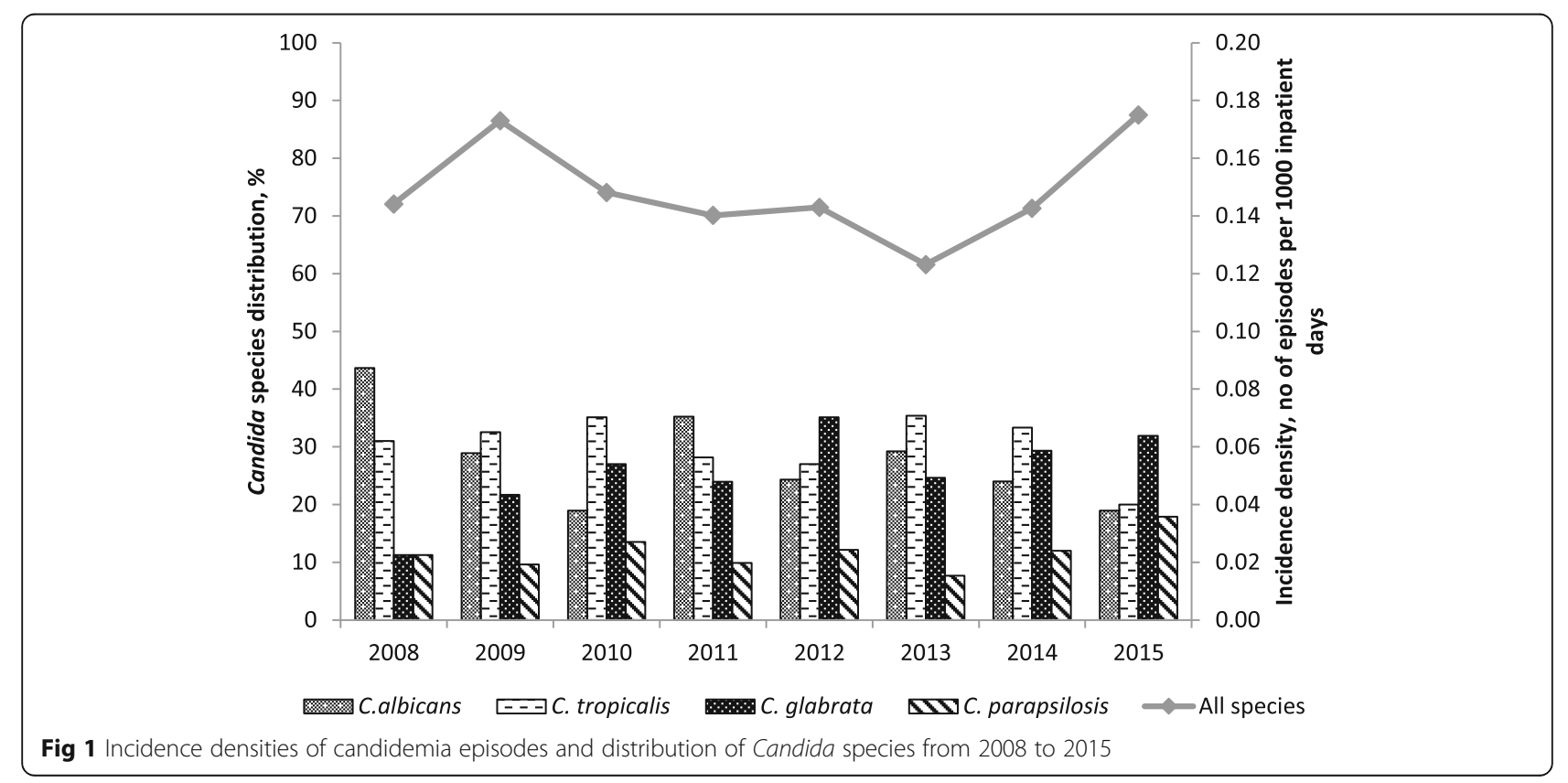


However, we did note that the overall incidence density increased from 0.14 in 2014 to 0.18 episodes/1000 inpatient-days in 2015, suggesting the need for continual monitoring. There was a significant increasing trend in the incidence density of C. glabrata (Coefficient 0.004, CI $0-0.007, p=0.04$ ), while the incidence densities of the other Candida spp. remained stable. The proportions of C. glabrata increased from $11.3 \%$ in 2008 to $31.6 \%$ in 2015 and that of C. albicans decreased from $44 \%$ in 2008 to $19 \%$ in 2015 .

\section{Antifungal susceptibilities}

Antifungal susceptibilities were available for 271 isolates, except for one $C$. parapsilosis (Table 1). Among isolates with available clinical breakpoints, overall susceptibility rates were $59.5 \%$ (153/257) for fluconazole, 86.9\% (152/ 175) for voriconazole, $99.2 \%$ (255/257) for anidulafungin, 98.1\% (252/257) for caspofungin and 98.9\% (254/257) for micafungin. Using the clinical breakpoints, C. albicans and $C$. parapsilosis retained high susceptibility $(>94 \%)$ to fluconazole and voriconazole. However, more than $20 \%$ of the C. tropicalis isolates were nonsusceptible to fluconazole and voriconazole. The proportions of isolates classified as wild-type (MIC value less than or equals to ECV) for fluconazole, voriconazole, itraconazole and posaconazole were similar among $C$. albicans, C. glabrata and C. parapsilosis (ranged from 94-100\%). Decreased susceptibilities (non wild-type; $\mathrm{MIC}$ value greater than ECV) to fluconazole and voriconazole were prominent in $C$. tropicalis isolates. Echinocandin resistance was rare, occurring only in three isolates $(C$. albicans $=1$; C. tropicalis $=1$ and C. glabrata $=1$ ) when assessed using both clinical breakpoints and ECVs. Most isolates had amphotericin B and flucytosine MICs below ECVs (96-100\%), although a number of $C$. parapsilosis were classified as non-wild-type (20\%). The amphotericin B MICs of these non-wild-type isolates were $2 \mu \mathrm{g} / \mathrm{mL}$, which were just one dilution above the ECV $(1 \mu \mathrm{g} / \mathrm{mL})$ utilised in this study. Furthermore, the ECV used in this study was derived using the YeastOne method and is one dilution lower than the ECVs for the other species $(2 \mu \mathrm{g} / \mathrm{mL})$ and the ECV derived from broth dilution methods.

$f k s$ mutations were detected in the echinocandinresistant C. albicans (caspofungin MIC $4 \mu \mathrm{g} / \mathrm{mL}$; anidulafungin MIC $0.25 \mu \mathrm{g} / \mathrm{mL}$; micafungin MIC $2 \mu \mathrm{g} / \mathrm{mL}$ ) and C. tropicalis (caspofungin MIC $2 \mu \mathrm{g} / \mathrm{mL}$; anidulafungin MIC $0.5 \mu \mathrm{g} / \mathrm{mL}$; micafungin $1 \mu \mathrm{g} / \mathrm{mL}$ ) isolates. Both isolates harboured a point mutation ( $6645 \mathrm{P}$ in $C$. albicans and S80P in C. tropicalis) in the hotspot 1 region of the $f k s 1$ gene. The two isolates remained susceptible to all other antifungals. Interestingly, $f k s$ mutations were not identified in the C. glabrata isolate which was resistant (caspofungin $\mathrm{MIC} \geq 8 \mu \mathrm{g} / \mathrm{mL}$; anidulafungin MIC $4 \mu \mathrm{g} / \mathrm{mL}$; micafungin MIC $4 \mu \mathrm{g} / \mathrm{mL}$ ).

\section{Clinical characteristics}

The clinical characteristics of the candidemia episodes are summarised in Table 2. The median age of patients with candidemia was 65 years and incidence did not differ by gender $(52.9 \%$ male $v s .47 .1 \%$ female, $p=0.59)$. The episodes occurred primarily in the medical wards (42.1\%), followed by intensive care units (ICUs) (38.3\%), surgical wards (19.5\%). Patients admitted to haematologyoncology (19.9\%), internal medicine (19.5\%) and general surgery units (12.3\%) encountered the most episodes.

Most of the patients presented with multiple comorbidities (median Charlson score $=5$, range 0-15), with many having malignancies (40.6\%). Diabetes was also common among these patients (39.5\%). Prior antibiotic exposure (90.4\%), central venous catheter placement (73.6\%), and surgery (65.1\%) were common risk factors. A large number of patients were colonised or infected with Candida at other non-blood sites (45.2\%) and had concurrent bacterial infections (48.7\%). In addition, it appears that candidemia episodes caused by $C$. tropicalis were more commonly encountered in patients with haematological malignancies $(p=0.01)$, neutropenia $(p<0.001)$ and higher SAPS II scores $(p=0.02)$. Exposure to echinocandins was also associated with candidemia episodes caused by C. parapsilosis $(p=0.001)$.

\section{Antifungal therapy and outcomes}

Antifungal therapy was initiated in 225 (86.2\%) episodes (Table 2). All but six of the 36 patients who did not receive treatment died before blood cultures flagged positive. Treatment was not initiated in four patients as they were conservatively managed. Interestingly, physicians elected not to initiate treatment in the remaining two patients.

Echinocandins were the initial treatment of choice (73.3\%), followed by azoles (23.1\%). Caspofungin (93.4\%) was more commonly used, since it was the only echinocandin in the formulary until anidulafungin's inclusion in August 2015. Among the patients receiving treatment, $32(14.2 \%)$ were already receiving antifungals as prophylaxis or empiric treatment on the day which cultures were taken. Fluconazole was the only azole used as initial treatment of candidemia in our institution. The median (range) time to initial treatment was 1 (0-9) days. Treatment was initiated in $73(32.4 \%)$ patients on day of culture and in $172(76.4 \%)$ patients within two days. The median (range) duration of therapy was 15 (1-140) days.

Patients with candidemia were moderately to severelyill - 57.9\% were having severe sepsis and the median (range) SAPS II score was $49(14-103)$ at the time of culture. Many of these episodes (38.3\%) occurred in 
Table 1 Antifungal susceptibilities of major species of Candida isolates ${ }^{\mathrm{a}}$

\begin{tabular}{|c|c|c|c|c|c|c|c|}
\hline Antifungal & $\begin{array}{l}\mathrm{MIC}_{50} \\
(\mu \mathrm{g} / \mathrm{mL})\end{array}$ & $\begin{array}{l}\mathrm{MIC}_{90} \\
(\mu \mathrm{g} / \mathrm{mL})\end{array}$ & $\begin{array}{l}\text { MIC Range } \\
(\mu \mathrm{g} / \mathrm{mL})\end{array}$ & $\% \mathrm{~S}^{\mathrm{b}}$ & $\% S D D / /^{b}$ & $\% R^{b}$ & $\% \mathrm{WT}^{c}$ \\
\hline \multicolumn{8}{|l|}{ Calbicans $(n=62)$} \\
\hline Fluconazole & 0.5 & 2 & $\leq 0.12->256$ & 95.2 & 1.6 & 3.2 & 93.5 \\
\hline Itraconazole & 0.06 & 0.12 & $\leq 0.015->16$ & - & - & - & 96.7 \\
\hline Posaconazole & 0.015 & 0.06 & $\leq 0.08->8$ & - & - & - & 96.7 \\
\hline Voriconazole & $\leq 0.008$ & 0.03 & $\leq 0.008->8$ & 93.6 & 3.2 & 3.2 & 93.5 \\
\hline Anidulafungin & $\leq 0.015$ & 0.03 & $\leq 0.015-0.25$ & 100 & 0 & 0 & 98.4 \\
\hline Caspofungin & 0.03 & 0.06 & $0.015-4$ & 98.4 & 0 & 1.6 & 98.4 \\
\hline Micafungin & $\leq 0.008$ & 0.015 & $\leq 0.008-2$ & 98.4 & 0 & 1.6 & 98.4 \\
\hline Flucytosine & $\leq 0.06$ & 0.25 & $\leq 0.06->64$ & - & - & - & 96.7 \\
\hline Amphotericin B & 0.5 & 1 & $\leq 0.12-1$ & - & - & - & 100 \\
\hline \multicolumn{8}{|l|}{ C. glabrata $(n=82)$} \\
\hline Fluconazole & 16 & 32 & $1->256$ & - & 92.8 & 7.2 & 97.6 \\
\hline Itraconazole & 1 & 1 & $0.12->16$ & - & - & - & 93.9 \\
\hline Posaconazole & 2 & 2 & $0.12->8$ & - & - & - & 95.1 \\
\hline Voriconazole & 0.5 & 2 & $0.03->8$ & - & - & - & 97.6 \\
\hline Anidulafungin & 0.03 & 0.06 & $\leq 0.015-4$ & 98.8 & 0 & 1.2 & 98.7 \\
\hline Caspofungin & 0.12 & 0.12 & $0.03->8$ & 96.4 & 2.4 & 1.2 & 96.3 \\
\hline Micafungin & 0.015 & 0.015 & $\leq 0.008-4$ & 98.8 & 0 & 1.2 & 98.7 \\
\hline Flucytosine & $\leq 0.06$ & 0.12 & $\leq 0.06-0.25$ & - & - & - & 100 \\
\hline Amphotericin B & 1 & 1 & $0.25-2$ & - & - & - & 100 \\
\hline \multicolumn{8}{|l|}{ C. tropicalis $(n=78)$} \\
\hline Fluconazole & 2 & 64 & $0.5->256$ & 78.2 & 5.1 & 16.7 & 84.6 \\
\hline Itraconazole & 0.25 & 0.5 & $0.03->16$ & - & - & - & 96.1 \\
\hline Posaconazole & 0.12 & 0.5 & $0.03-4$ & - & - & - & 98.7 \\
\hline Voriconazole & 0.12 & 4 & $\leq 0.008->8$ & 75.6 & 11.5 & 12.8 & 80.8 \\
\hline Anidulafungin & 0.03 & 0.12 & $\leq 0.015-0.5$ & 98.7 & 1.3 & 0 & 98.7 \\
\hline Caspofungin & 0.03 & 0.06 & $0.015-2$ & 98.7 & 0 & 1.3 & 98.7 \\
\hline Micafungin & 0.03 & 0.03 & $\leq 0.008-1$ & 98.7 & 0 & 1.3 & 98.7 \\
\hline Flucytosine & $\leq 0.06$ & 0.12 & $\leq 0.06-32$ & - & - & - & 96.2 \\
\hline Amphotericin B & 1 & 1 & $0.25-2$ & - & - & - & 100 \\
\hline \multicolumn{8}{|c|}{ C. parapsilosis $(n=35)$} \\
\hline Fluconazole & 0.5 & 2 & $0.25-4$ & 97.1 & 2.9 & 0 & 100 \\
\hline Itraconazole & 0.06 & 0.06 & $\leq 0.015-0.12$ & - & - & - & 100 \\
\hline Posaconazole & 0.03 & 0.06 & $0.015-0.12$ & - & - & - & 100 \\
\hline Voriconazole & 0.015 & 0.03 & $\leq 0.008-0.6$ & 100 & 0 & 0 & 97.1 \\
\hline Anidulafungin & 0.5 & 2 & $0.12-2$ & 100 & 0 & 0 & 100 \\
\hline Caspofungin & 0.25 & 0.5 & $0.06-1$ & 100 & 0 & 0 & 100 \\
\hline Micafungin & 0.5 & 2 & $0.12-2$ & 100 & 0 & 0 & 100 \\
\hline Flucytosine & $\leq 0.06$ & 0.5 & $\leq 0.06-1$ & - & - & - & 100 \\
\hline Amphotericin B & 1 & 2 & $0.25-2$ & - & - & - & 80.0 \\
\hline
\end{tabular}

$S$ susceptible, SDD susceptible dose-dependent, I intermediate, $R$ resistant, $W T$ wild-type

${ }^{a} \mathrm{MICs}$ are only reflected for the predominant species

${ }^{b}$ Susceptibilities were assessed based on CLSI species-specific clinical interpretative breakpoints [12]. Clinical breakpoints are not available for itraconazole, posaconazole, flucytosine and amphotericin B for all species and voriconazole for C. glabrata

'ECVs were derived from $[13,14]$ and $[15]$ 
Table 2 Clinical characteristics of candidemia episodes

\begin{tabular}{|c|c|c|c|c|c|c|}
\hline & All & C. glabrata & C. tropicalis & C. albicans & C. parapsilosis & $p$ \\
\hline & $n=261$ & $n=75$ (28.6\%) & $n=71(27.1 \%)$ & $n=59(22.6 \%)$ & $n=33(12.6 \%)$ & \\
\hline \multicolumn{7}{|l|}{ Demographics } \\
\hline Male sex & $138(52.9)$ & $37(49.3)$ & $39(54.9)$ & $32(54.2)$ & $22(66.7)$ & 0.42 \\
\hline Median age (range) & $65(22-101)$ & $67(24-95)$ & $63(28-90)$ & $68(27-101)$ & $61(28-86)$ & 0.06 \\
\hline Ward type & & & & & & 0.83 \\
\hline Medical ward & $110(42.1)$ & $30(40.0)$ & $35(49.3)$ & $23(39.0)$ & $14(42.4)$ & \\
\hline Surgical ward & $51(19.5)$ & $16(21.3)$ & $10(14.1)$ & $14(23.7)$ & $7(21.2)$ & \\
\hline $\mathrm{ICU}$ & $100(38.3)$ & $29(38.7)$ & 26 (36.6) & $22(37.3)$ & $12(36.4)$ & \\
\hline Elective admission & $27(10.3)$ & $12(16.0)$ & $5(7.0)$ & $5(8.5)$ & $4(12.1)$ & 0.32 \\
\hline \multicolumn{7}{|l|}{ Comorbidities } \\
\hline Malignancies & $106(40.6)$ & $34(45.3)$ & $29(40.8)$ & $23(39.0)$ & $12(36.4)$ & 0.81 \\
\hline Haematological & $27(10.3)$ & $3(4.0)$ & $13(18.3)$ & $6(10.2)$ & $2(6.1)$ & 0.03 \\
\hline Oncological & $84(32.2)$ & $32(42.7)$ & $17(23.9)$ & $18(30.5)$ & $11(33.3)$ & 0.11 \\
\hline With metastases & $36(13.8)$ & $16(21.3)$ & $11(15.5)$ & $6(10.2)$ & $3(9.1)$ & 0.23 \\
\hline Diabetes & $103(39.5)$ & $31(41.3)$ & $25(35.2)$ & $23(39.0)$ & $12(36.4)$ & 0.89 \\
\hline Chronic renal failure & $67(25.7)$ & $17(22.7)$ & $22(31.0)$ & $14(23.7)$ & $8(24.2)$ & 0.67 \\
\hline Hepatobiliary disorders & $58(22.2)$ & $17(22.7)$ & $20(28.2)$ & $8(13.6)$ & $8(24.2)$ & 0.25 \\
\hline Myocardial infarction & $43(16.5)$ & $10(13.3)$ & $13(18.3)$ & $15(25.4)$ & $1(3.0)$ & 0.04 \\
\hline Cerebrovascular disease & $29(11.1)$ & $12(16.0)$ & $8(11.3)$ & $4(6.8)$ & $4(12.1)$ & 0.44 \\
\hline Median (range) Charlson score & $5(0-15)$ & $6(0-15)$ & $5(0-14)$ & $4(0-12)$ & $4(0-9)$ & 0.08 \\
\hline \multicolumn{7}{|l|}{ Risk factors } \\
\hline Central venous catheter & $192(73.6)$ & $47(62.7)$ & $55(77.5)$ & $46(78.0)$ & $26(78.8)$ & 0.11 \\
\hline Drain & $60(23.0)$ & $22(29.3)$ & $14(19.7)$ & $16(27.1)$ & $6(18.2)$ & 0.43 \\
\hline Mechanical ventilation & $111(42.5)$ & $26(34.7)$ & $31(43.7)$ & $25(42.4)$ & $16(48.5)$ & 0.52 \\
\hline Total parenteral nutrition & $52(19.9)$ & $12(16.0)$ & $13(18.3)$ & $12(20.3)$ & $10(30.3)$ & 0.37 \\
\hline Surgery & $170(65.1)$ & $51(68.0)$ & $44(66.0)$ & $39(66.1)$ & $20(60.6)$ & 0.83 \\
\hline Gastrointestinal surgery & $41(15.7)$ & $18(24.0)$ & $5(7.0)$ & $9(24.3)$ & $5(15.2)$ & 0.05 \\
\hline Renal replacement therapy & $85(32.6)$ & $16(21.3)$ & $28(39.4)$ & $21(35.6)$ & $12(36.4)$ & 0.10 \\
\hline Antimicrobial therapy & $236(90.4)$ & $67(89.3)$ & $68(95.8)$ & $53(89.8)$ & $27(81.8)$ & 0.15 \\
\hline Antifungal therapy & $51(19.5)$ & $13(17.3)$ & $15(21.1)$ & $8(13.6)$ & $11(33.3)$ & 0.13 \\
\hline Azole & $24(9.2)$ & $5(6.7)$ & $10(14.1)$ & $6(10.2)$ & $2(6.1)$ & 0.41 \\
\hline Echinocandin & $30(11.5)$ & $8(10.7)$ & $6(8.5)$ & $2(3.4)$ & $10(30.3)$ & 0.001 \\
\hline Immunosuppressive therapy & $76(29.1)$ & $17(22.7)$ & $28(39.4)$ & $16(27.1)$ & $10(30.3)$ & 0.16 \\
\hline Neutropenia & $21(8.0)$ & $3(4.0)$ & $13(18.3)$ & $2(3.4)$ & $2(6.1)$ & 0.004 \\
\hline \multicolumn{7}{|l|}{ Therapy } \\
\hline Primary therapy & & & & & & 0.15 \\
\hline Echinocandin & $165(73.3)$ & $45(76.3)$ & $49(81.7)$ & $32(60.4)$ & $22(71.0)$ & \\
\hline Azole & $52(23.1)$ & $12(20.3)$ & $11(18.3)$ & $17(32.0)$ & $8(25.8)$ & \\
\hline Others & $8(3.1)$ & $2(3.3)$ & $1(1.7)$ & $4(6.8)$ & $1(3.2)$ & \\
\hline None & $36(13.8)$ & $16(21.3)$ & $10(14.1)$ & $6(10.2)$ & $2(6.1)$ & \\
\hline $\begin{array}{l}\text { Median (range) time to primary } \\
\text { therapy, days }\end{array}$ & $1(0-9)$ & $2(0-7)$ & $1(0-3)$ & $2(0-5)$ & $1(0-9)$ & 0.01 \\
\hline $\begin{array}{l}\text { Median (range) duration of } \\
\text { therapy, days }\end{array}$ & $15(1-140)$ & $16(2-61)$ & $11(1-96)$ & $16(1-140)$ & $15(2-47)$ & 0.01 \\
\hline
\end{tabular}


Table 2 Clinical characteristics of candidemia episodes (Continued)

\begin{tabular}{|c|c|c|c|c|c|c|}
\hline \multicolumn{7}{|l|}{ Infection Characteristics \& Outcomes } \\
\hline $\begin{array}{l}\text { Median (range) time to positive } \\
\text { culture, days }\end{array}$ & $12(0-282)$ & $11(0-282)$ & $14(0-123)$ & $14(0-79)$ & $37(0-104)$ & 0.37 \\
\hline $\begin{array}{l}\text { Median (range) time to reporting } \\
\text { positive culture, days }\end{array}$ & $2(0-10)$ & $3(0-9)$ & $1(0-10)$ & $2(1-5)$ & $2(1-3)$ & $<0.001$ \\
\hline $\begin{array}{l}\text { Median (range) time to species } \\
\text { identification, days }\end{array}$ & $5(2-22)$ & $6(2-12)$ & $4(2-12)$ & $5(2-9)$ & $5(3-7)$ & $<0.001$ \\
\hline Median (range) SAPS II score & $49(14-103)$ & $48(18-95)$ & $55(18-93)$ & $48(23-103)$ & $44(14-72)$ & 0.01 \\
\hline $\begin{array}{l}\text { Median (range) Pitts' } \\
\text { bacteraemia score }\end{array}$ & $3(0-14)$ & $2(0-11)$ & $3(0-12)$ & $3(0-11)$ & $2(0-8)$ & 0.86 \\
\hline Severe sepsis at time of culture & $151(57.9)$ & $49(65.3)$ & $43(60.6)$ & $33(55.9)$ & $13(58.0)$ & 0.08 \\
\hline ICU stay & $131(50.2)$ & $36(48.0)$ & $35(49.3)$ & $30(50.8)$ & $16(48.5)$ & 0.99 \\
\hline Concurrent infection & $127(48.7)$ & $33(44.0)$ & $36(57.0)$ & $30(50.8)$ & $16(48.5)$ & 0.83 \\
\hline $\begin{array}{l}\text { Candida colonization/infection } \\
\text { at other sites }\end{array}$ & $118(45.2)$ & $41(54.7)$ & $39(54.9)$ & $34(57.6)$ & $18(54.5)$ & 0.99 \\
\hline $\begin{array}{l}\text { 30-day in-hospital all-cause } \\
\text { mortality }\end{array}$ & $130(49.8)$ & $38(50.7)$ & $42(59.2)$ & $28(47.5)$ & $9(27.3)$ & 0.03 \\
\hline
\end{tabular}

All variables are denoted as number of patients with the characteristic or belonging to the category [n (\%)], unless otherwise stated

Sub-group analyses are shown only for episodes involving major Candida spp. and not for mixed candidemia and less common species

Comorbidities $<10 \%$ in occurrence are not reflected

Significant variables are reflected in bold and italics

critically-ill patients warded in the ICUs. We also observed that some patients (11.9\%), who were initially in the general wards at the time of culture, required admission into the ICU after Candida isolation, suggesting that candidemia episodes can result in severe illness. Mortality occurred in 150 (57.4\%) episodes during the admission. The 7-day, 14-day and 30-day in-hospital mortality rates were $28.3 \%, 39.8 \%$, and $49.8 \%$. The mortality rate was lowest in patients infected with $C$. parapsilosis $(23.5 \%)(p=0.03)$. Among the 225 patients who received treatment, the 30-day in-hospital mortality rate was $41.4 \%$, while all but two $(94.4 \%)$ of the nontreated episodes resulted in death.

\section{Predictors of mortality}

The characteristics of survivors and non-survivors at 30 days are depicted in Table 3. Based on the multivariable logistic regression model, high SAPS II score (Odds ratio, OR 1.08; 95\% confidence interval, CI 1.06-1.11) and renal replacement therapy (OR 4.31; CI 2.24-8.28) were the only factors associated with 30 -day mortality. Presence of drains was a protective factor (OR 0.45; CI 0.21-0.94). Mortality occurred rapidly in many of the non-survivors, hence receipt/type of antifungal therapy was not included in this model, since antifungal therapy could not be initiated in this subset of patients. To examine the impact of initial antifungal therapy on 30-day mortality, a separate analysis was performed for candidemia episodes where treatment was administered. Results were similar when non-treated episodes were excluded. High SAPS II score, renal replacement therapy and drains placement were significant factors in the multivariable regression model (Table 4). The choice and timing of initial antifungal therapy was not associated with mortality.

\section{Discussion}

We report here a comprehensive epidemiological study of candidemia conducted at a large tertiary regional referral centre, which included the clinical characteristics, antifungal treatment, species distribution, antifungal susceptibilities and outcomes of candidemia. Our study showed that the incidence density of candidemia in our institution has remained fairly stable since 2008. This concurs with the general trend of stability in incidence reported in other developed countries, such as the United States and Europe [2, 17]. A recent study comparing candidemias among sites in Asia indicated that rates in Singapore (0.15 episodes per 1000 patient-days) were comparable with most other Asian countries, with the exception of Taiwan (0.37 per 1000 patient-days) and India (1.24 per 1000 patient-days) [10]. On a more global scale, our rates were lower than those in Italy $(0.33$ per 1000 patient-days) [18], and Brazil (0.37 per 1000 patient-days) [19]. It appears that the species distribution in our institution is changing. Previous local studies reported a predominance of C. tropicalis, a finding commonly observed in tropical regions $[10,20]$. We observed an increasing proportion of C. glabrata from $11 \%$ in 2008 to $31 \%$ in 2015 , overtaking C. tropicalis as the predominant species. 
Table 3 Characteristics of survivors vs. non-survivors

\begin{tabular}{|c|c|c|c|}
\hline & Survivors & Non-survivors & $p$ \\
\hline & $n=134$ & $n=127$ & \\
\hline \multicolumn{4}{|l|}{ Demographics } \\
\hline Male sex & $73(54.5)$ & $65(51.2)$ & 0.59 \\
\hline Median age (range) & $64(22-95)$ & $65(24-101)$ & 0.81 \\
\hline Ward type & & & $<0.001^{a}$ \\
\hline Medical ward & $66(49.3)$ & $44(34.6)$ & \\
\hline Surgical ward & $37(27.6)$ & $14(11.0)$ & \\
\hline $\mathrm{ICU}$ & $31(23.1)$ & $69(54.3)$ & \\
\hline Elective admission & $14(10.4)$ & $13(10.2)$ & 0.96 \\
\hline \multicolumn{4}{|l|}{ Comorbidities } \\
\hline Malignancies & $58(43.3)$ & $48(51.6)$ & 0.37 \\
\hline Diabetes & $53(39.6)$ & $50(39.4)$ & 0.97 \\
\hline Chronic renal failure & $22(16.4)$ & $45(35.4)$ & $<0.001$ \\
\hline Hepatobiliary disorders & $25(18.7)$ & $33(26.0)$ & 0.16 \\
\hline Myocardial infarction & $19(14.2)$ & $24(18.9)$ & 0.30 \\
\hline Cerebrovascular disease & $11(8.2)$ & $18(14.2)$ & 0.13 \\
\hline Median (range) Charlson score & $4(0-15)$ & $5(0-14)$ & $0.09^{\mathrm{a}}$ \\
\hline Median (range) SAPS II score & $43(14-82)$ & $58(27-103)$ & $<0.001^{\mathrm{a}}$ \\
\hline \multicolumn{4}{|l|}{ Risk factors } \\
\hline Central venous catheter & $89(66.4)$ & $103(81.1)$ & $0.007^{a}$ \\
\hline Drain & $37(27.6)$ & $23(18.1)$ & $0.07^{\mathrm{a}}$ \\
\hline Mechanical ventilation & $47(35.1)$ & $64(50.4)$ & $0.01^{\mathrm{a}}$ \\
\hline Total parenteral nutrition & $28(20.9)$ & $24(18.9)$ & 0.69 \\
\hline Surgery & $81(60.4)$ & $89(70.1)$ & 0.10 \\
\hline Gastrointestinal surgery & $20(14.9)$ & $21(16.5)$ & 0.72 \\
\hline Renal replacement therapy & $23(17.2)$ & $62(48.8)$ & $<0.001^{\mathrm{a}}$ \\
\hline Antimicrobial therapy & $116(86.6)$ & $120(94.5)$ & 0.30 \\
\hline Antifungal therapy & $27(20.1)$ & $24(18.9)$ & 0.79 \\
\hline Immunosuppressive therapy & $33(24.6)$ & $43(33.9)$ & 0.10 \\
\hline Neutropenia & $10(7.5)$ & $11(8.7)$ & 0.72 \\
\hline \multicolumn{4}{|l|}{ Therapy } \\
\hline Initial therapy & & & $<0.001^{b}$ \\
\hline Echinocandin & $89(66.4)$ & $76(59.8)$ & \\
\hline Azole & $40(29.9)$ & $12(9.4)$ & \\
\hline Others (Amphotericin or combination) & $3(2.2)$ & $5(3.9)$ & \\
\hline None & $2(1.5)$ & $34(26.8)$ & \\
\hline Received initial therapy within $24 \mathrm{~h}$ & $58(43.2)$ & $64(50.4)$ & $<0.001^{b}$ \\
\hline \multicolumn{4}{|l|}{ Infection Characteristics } \\
\hline Species & & & $0.04^{\mathrm{a}}$ \\
\hline C. albicans & $32(23.9)$ & $27(21.3)$ & \\
\hline C. glabrata & $39(29.1)$ & $36(28.3)$ & \\
\hline C. tropicalis & $29(21.6)$ & $42(33.1$ & \\
\hline C. parapsilosis & $24(17.9)$ & $9(7.1)$ & \\
\hline
\end{tabular}


Table 3 Characteristics of survivors vs. non-survivors (Continued)

\begin{tabular}{|c|c|c|c|}
\hline Median (range) time to reporting positive culture, days & $2(0-10)$ & $2(0-10)$ & $0.08^{\mathrm{a}}$ \\
\hline Median (range) time to species identification, days & $5(2-16)$ & $5(2-22)$ & $0.001^{\mathrm{a}}$ \\
\hline Median (range) Candida score & $2(0-5)$ & $3(0-5)$ & 0.01 \\
\hline Median (range) Pitts' bacteraemia score & $2(0-11)$ & $5(0-14)$ & $<0.001^{\mathrm{a}}$ \\
\hline Severe sepsis at time of culture & $64(47.8)$ & $87(68.5)$ & $0.001^{\mathrm{a}}$ \\
\hline Concurrent bacterial infection & $59(46.5)$ & $68(53.5)$ & 0.12 \\
\hline Candida colonization/infection at other sites & $61(45.5)$ & $57(44.9)$ & 0.92 \\
\hline
\end{tabular}

All variables are denoted as number of patients with the characteristic or belong to the category $n(\%)$, unless otherwise stated Significant variables are reflected in bold and italics

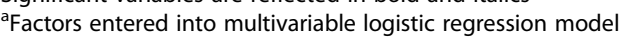

${ }^{b}$ Additional factors entered into multivariable logistic regression model including only treated episodes

With respect to antifungal susceptibilities, while $C$. albicans and C. parapsilosis remained mostly susceptible, fluconazole resistant rates of C. tropicalis was $17 \%$. Notably, the fluconazole $\mathrm{MIC}_{90}$ of C. tropicalis increased from $2 \mu \mathrm{g} / \mathrm{mL}$ in 2007 to $64 \mu \mathrm{g} / \mathrm{mL}$ reported in our study [20]. This MIC uptrend suggests that C. tropicalis, one of the predominant species in our context, is increasingly becoming less susceptible. Further molecular investigations are underway to understand the mechanisms related to azole resistance in these isolates.

Another noteworthy finding of our study was the emergence of echinocandin resistance in the Southeast Asia region. In the post-echinocandin era, there have been increasing reports of echinocandin treatment failures in most clinically-relevant species, especially in $C$. glabrata [7, 21-24]. Fortunately, resistance rates remained rare in the local context. There were only three (1.1\%) isolates which were echinocandin-resistant, of which two had $f k s$ mutations. To the best of our knowledge, this is the first incidence of $f k s$ mutations in Candida bloodstream isolates other than C. glabrata identified locally. While the $f k s$ mutations identified in our isolates have been previously described, it is interesting to note that resistance developed rapidly (within 4 days of exposure to caspofungin) in one of the patients. Development in resistance has been primarily related to prolonged use of echinocandins, which was observed in the other patient, who had received 30 days of caspofungin prior to Candida isolation [22].

Our study observed a high 30-day mortality rate of $49 \%$. Like many previous studies, we found that mortality was associated with severity of illness at onset of

Table 4 Multivariable logistic regression model for mortality in treated cases $(n=225)$

\begin{tabular}{ll}
\hline Variable & OR (95\% Cl) \\
\hline SAPS II score & $1.08(1.05-1.11)$ \\
Presence of drains & $0.44(0.19-0.99)$ \\
Renal replacement therapy & $5.54(2.80-10.97)$ \\
\hline
\end{tabular}

candidemia, suggesting that the poor outcomes of patients with candidemia is likely related to the poor prognosis of these patients with multiple comorbidities [25]. Receipt of renal replacement therapy was also associated with 30-day mortality. This could be an indication of the underlying organ dysfunction contributing to severity of illness. Drains placement prior to Candida isolation was found to be protective, suggesting that perhaps source control could contribute to better survival in patients with secondary candidemia.

Initial antifungal choice did not appear to be associated with mortality in our study. Although the Infectious Diseases Society of America guidelines have recommended the use of an echinocandin as a first-line agent, randomised controlled trials conducted so far have yet to conclusively demonstrate superiority of one agent over another [26-28]. A recent study has also illustrated that clinical severity, rather than initial antifungal strategy, was significantly correlated with mortality [25]. One reason why we were unable to detect any association of initial antifungal choice with mortality could be because we did not account for the appropriateness of the therapy in terms of dosing. Furthermore, pharmacokinetic variability can result in fluctuating antifungal levels in individual patients [29]. Perhaps, the impact of initial antifungal choice on treatment outcomes can be better elucidated if antifungal dosing was individualised, such as through the use of therapeutic drug monitoring. This therapeutic approach is currently being explored in our institution.

Although a large number of our patients received antifungals in a timely fashion, there was still a delay in therapy for some patients, with some receiving antifungals more than a week after cultures were taken. The time to administration of antifungals could be limited by the lack of rapid diagnostic tests available in our institution. It takes an average of two days to report a positive Candida blood culture, and in some instances even up to a week.

Our study was not without limitations. This was a single-centre study and our results might not be extrapolated to other institutions as the epidemiology of 
candidemia can be highly institution-specific. The retrospective nature of the study also precluded the analysis of impact of time of catheter removal on mortality. Nevertheless, this study provides important epidemiological findings which are instrumental in designing strategies for better management of candidemia in our institution.

\section{Conclusions}

While incidence of candidemia appeared to be stable, incidence of C. glabrata is increasing. C. glabrata and C. tropicalis contributed to majority of the candidemia cases in our institution. Decreasing azole susceptibilities to $C$. tropicalis and the emergence of echinocandin resistance suggests that susceptibility patterns may no longer be sufficiently predicted by speciation in our institution. Routine antifungal susceptibility, particularly for C. tropicalis, might be essential to guide clinician to effectively manage patients with invasive Candida infections. Candidemia was associated with high mortality, and antifungal stewardship efforts in individualising antifungal dosing through therapeutic drug monitoring should be further explored to improve outcomes in this population.

\section{Abbreviations \\ ATCC: American Type Culture Collection; Cl: Confidence interval; CLSI: Clinical and Laboratory Standards Institute; ECV: Epidemiological cut-off values; ICU: Intensive care unit; MIC: Minimum inhibitory concentration; OR: Odds ratio; PCR: Polymerase chain reaction; SAPS: Simplified acute physiology score; SGH: Singapore General Hospital}

\section{Acknowledgements}

The authors acknowledge the excellent assistance of lab members of the Pathology Lab, in particular Ms Tan Mei Gie, and Pharmacy Research Lab, Singapore, in the collection of the isolates.

\section{Funding}

This study was funded by grants from National Medical Research Council (NMRC/TA/0025/2013 and NMRC/CG/016/2013); SGH Supplementary Research Grant (SRG \#15/20); and Pfizer Inc. (WS2347894). The grant agencies had no involvement in the study design, in the collection, analysis and interpretation of the data, or in the decision to submit the article for publication.

\section{Availability of data and materials}

Please contact author for data requests.

\section{Authors' contributions}

JQT, SRC, SJL, SYC, HL, TPL and ALT participated in the microbiological and/ or molecular experiments. JQT, SRC, HPN, KWL, YC and WL collected clinical data. SRC prepared the initial draft of the manuscript. JQT and ALK conceived the study, interpreted the results, revised the manuscript and wrote the manuscript. RPE participated in the design of the study and revised the manuscript. All authors read and approved the final manuscript.

\section{Competing interests}

The authors declare that they have no competing interests.

\section{Consent for publication}

Not applicable.

\section{Ethics approval and consent to participate}

The research protocol was approved by the Singhealth Centralised Institutional Review Board (2013/987/D). Informed consent was waived in view of retrospective nature of study.

\section{Author details}

'Department of Pharmacy, Singapore General Hospital, Blk 8 Level 2, Outram Road, Singapore 169608, Singapore. ${ }^{2}$ Department of Microbiology, Singapore General Hospital, Outram Road, Singapore 169608, Singapore. ${ }^{3}$ Department of Pharmacy, National University of Singapore, 18 Science Drive 4, Singapore 117543, Singapore. ${ }^{4}$ SingHealth Duke-NUS Medicine Academic Clinical Programme, 20 College Rd, Singapore 169856, Singapore. ${ }^{5}$ Emerging Infectious Diseases, Duke-NUS Medical School, 8 College Rd, Singapore 169857, Singapore. ${ }^{6}$ Present address: Tan Tock Seng Hospital, 11 Jalan Tan Tock Seng, Singapore 308433, Singapore.

Received: 22 August 2016 Accepted: 16 February 2017

Published online: 11 March 2017

\section{References}

1. Magill SS, Edwards JR, Bamberg W, Beldavs ZG, Dumyati G, Kainer MA, Lynfield R, Maloney M, McAllister-Hollod L, Nadle J, Ray SM, Thompson DL, Wilson LE, Fridkin SK. Emerging Infections Program Healthcare-Associated I, Antimicrobial Use Prevalence Survey T. Multistate point-prevalence survey of health care-associated infections. N Engl J Med. 2014;370(13):1198-208.

2. Bassetti M, Merelli M, Ansaldi F, de Florentiis D, Sartor A, Scarparo C, Callegari A, Righi E. Clinical and therapeutic aspects of candidemia: a five year single centre study. PLoS One. 2015;10(5):e0127534.

3. Pfaller M, Neofytos D, Diekema D, Azie N, Meier-Kriesche HU, Quan SP, Horn D. Epidemiology and outcomes of candidemia in 3648 patients: data from the Prospective Antifungal Therapy (PATH Alliance(R)) registry, 2004-2008. Diagn Microbiol Infect Dis. 2012;74(4):323-31.

4. Diekema D, Arbefeville S, Boyken L, Kroeger J, Pfaller M. The changing epidemiology of healthcare-associated candidemia over three decades. Diagn Microbiol Infect Dis. 2012;73(1):45-8.

5. Hassan I, Powell G, Sidhu M, Hart WM, Denning DW. Excess mortality, length of stay and cost attributable to candidaemia. J Infect. 2009;59(5):360-5.

6. Falagas ME, Apostolou KE, Pappas VD. Attributable mortality of candidemia: a systematic review of matched cohort and case-control studies. Eur J Clin Microbiol Infect Dis. 2006;25(7):419-25.

7. Alexander $B D$, Johnson MD, Pfeiffer CD, Jimenez-Ortigosa C, Catania J, Booker R, Castanheira M, Messer SA, Perlin DS, Pfaller MA. Increasing echinocandin resistance in Candida glabrata: clinical failure correlates with presence of FKS mutations and elevated minimum inhibitory concentrations. Clin Infect Dis. 2013;56(12):1724-32.

8. Montagna MT, Lovero G, Borghi E, Amato G, Andreoni S, Campion L, Lo Cascio G, Lombardi G, Luzzaro F, Manso E, Mussap M, Pecile P, Perin S, Tangorra E, Tronci M, latta R, Morace G. Candidemia in intensive care unit: a nationwide prospective observational survey (GISIA-3 study) and review of the European literature from 2000 through 2013. Eur Rev Med Pharmacol Sci. 2014;18(5):661-74.

9. Guinea J. Global trends in the distribution of Candida species causing candidemia. Clin Microbiol Infect. 2014;20 Suppl 6:5-10.

10. Tan BH, Chakrabarti A, Li RY, Patel AK, Watcharananan SP, Liu Z, Chindamporn A, Tan AL, Sun PL, Wu UI, Chen YC. Asia Fungal Working G. Incidence and species distribution of candidaemia in Asia: a laboratorybased surveillance study. Clin Microbiol Infect. 2015;21(10):946-53.

11. Tan TY, Hsu LY, Alejandria MM, Chaiwarith R, Chinniah T, Chayakulkeeree M, Choudhury S, Chen YH, Shin JH, Kiratisin P, Mendoza M, Prabhu K, Supparatpinyo K, Tan AL, Phan XT, Tran TT, Nguyen GB, Doan MP, Huynh VA, Nguyen SM, Tran TB, Van Pham H. Antifungal susceptibility of invasive Candida bloodstream isolates from the Asia-Pacific region. Med Mycol. 2016;54(5):471-7.

12. CLSI. Reference Method for Broth Dilution Antifungal Susceptibility Testing of Yeasts; Fourth Informational Supplement. CLSI document M27-S4. Wayne: Clinical and Laboratory Standards Institute; 2012.

13. Canton E, Peman J, Hervas D, Iniguez C, Navarro D, Echeverria J, MartinezAlarcon J, Fontanals D, Gomila-Sard B, Buendia B, Torroba L, Ayats J, Bratos A, Sanchez-Reus F, Fernandez-Natal I. Comparison of three statistical methods for establishing tentative wild-type population and epidemiological cutoff values for echinocandins, amphotericin B, flucytosine, and six Candida species as determined by the colorimetric Sensititre YeastOne method. J Clin Microbiol. 2012;50(12):3921-6.

14. Canton E, Peman J, Iniguez C, Hervas D, Lopez-Hontangas JL, Pina-Vaz C, Camarena JJ, Campos-Herrero I, Garcia-Garcia I, Garcia-Tapia AM, Guna R, Merino P, Perez del Molino L, Rubio C, Suarez A, Group FS. Epidemiological 
cutoff values for fluconazole, itraconazole, posaconazole, and voriconazole for six Candida species as determined by the colorimetric Sensititre YeastOne method. J Clin Microbiol. 2013;51(8):2691-5.

15. Espinel-Ingroff A, Alvarez-Fernandez M, Canton E, Carver PL, Chen SC, Eschenauer G, Getsinger DL, Gonzalez GM, Govender NP, Grancini A, Hanson KE, Kidd SE, Klinker K, Kubin CJ, Kus JV, Lockhart SR, Meletiadis J, Morris AJ, Pelaez T, Quindos G, Rodriguez-Iglesias M, Sanchez-Reus F, Shoham S, Wengenack NL, Borrell Sole N, Echeverria J, Esperalba J, Gomez GPE, Garcia Garcia I, Linares MJ, Marco F, Merino P, Peman J, Perez Del Molino L, Rosello Mayans E, Rubio Calvo C, Ruiz Perez de Pipaon M, Yague G, Garcia-Effron G, Guinea J, Perlin DS, Sanguinetti M, Shields R, Turnidge J. Multicenter study of epidemiological cutoff values and detection of resistance in Candida spp. to anidulafungin, caspofungin, and micafungin using the Sensititre YeastOne colorimetric method. Antimicrob Agents Chemother. 2015;59(11):6725-32.

16. Desnos-Ollivier M, Bretagne S, Raoux D, Hoinard D, Dromer F, Dannaoui E. Mutations in the fks 1 gene in Candida albicans, C. tropicalis, and C. krusei correlate with elevated caspofungin MICs uncovered in AM3 medium using the method of the European Committee on Antibiotic Susceptibility Testing. Antimicrob Agents Chemother. 2008;52(9):3092-8.

17. Cleveland AA, Harrison LH, Farley MM, Hollick R, Stein B, Chiller TM, Lockhart SR, Park BJ. Declining incidence of candidemia and the shifting epidemiology of Candida resistance in two US metropolitan areas, 2008-2013: results from population-based surveillance. PLoS One. 2015;10(3):e0120452.

18. Posteraro B, Spanu T, Fiori B, De Maio F, De Carolis E, Giaquinto A, Prete V, De Angelis G, Torelli R, D'Inzeo T, Vella A, De Luca A, Tumbarello M, Ricciardi W, Sanguinetti M. Antifungal Susceptibility Profiles of Bloodstream Yeast Isolates by Sensititre YeastOne over Nine Years at a Large Italian Teaching Hospital. Antimicrob Agents Chemother. 2015;59(7):3944-55.

19. Colombo AL, Nucci M, Park BJ, Nouer SA, Arthington-Skaggs B, da Matta DA, Warnock D, Morgan J. Brazilian Network Candidemia S. Epidemiology of candidemia in Brazil: a nationwide sentinel surveillance of candidemia in eleven medical centers. J Clin Microbiol. 2006;44(8):2816-23.

20. Tan TY, Tan AL, Tee NW, Ng LS, Chee CW. The increased role of nonalbicans species in candidaemia: results from a 3-year surveillance study. Mycoses. 2010;53(6):515-21.

21. Ruggero MA, Topal JE. Development of echinocandin-resistant Candida albicans candidemia following brief prophylactic exposure to micafungin therapy. Transpl Infect Dis. 2014;16(3):469-72.

22. Jensen RH, Justesen US, Rewes A, Perlin DS, Arendrup MC. Echinocandin failure case due to a previously unreported FKS1 mutation in Candida krusei. Antimicrob Agents Chemother. 2014;58(6):3550-2.

23. Pfeiffer CD, Garcia-Effron G, Zaas AK, Perfect JR, Perlin DS, Alexander BD. Breakthrough invasive candidiasis in patients on micafungin. J Clin Microbiol. 2010:48(7):2373-80

24. Garcia-Effron G, Kontoyiannis DP, Lewis RE, Perlin DS. Caspofungin-resistant Candida tropicalis strains causing breakthrough fungemia in patients at high risk for hematologic malignancies. Antimicrob Agents Chemother. 2008;52(11):4181-3.

25. Murri R, Scoppettuolo G, Ventura G, Fabbiani M, Giovannenze F, Taccari F, Milozzi E, Posteraro B, Sanguinetti M, Cauda R, Fantoni M. Initial antifungal strategy does not correlate with mortality in patients with candidemia. Eur J Clin Microbiol Infect Dis. 2016;35(2):187-93.

26. Pappas PG, Kauffman CA, Andes DR, Clancy CJ, Marr KA, Ostrosky-Zeichner L, Reboli AC, Schuster MG, Vazquez JA, Walsh TJ, Zaoutis TE, Sobel JD. Clinical Practice Guideline for the Management of Candidiasis: 2016 Update by the Infectious Diseases Society of America. Clin Infect Dis. 2016;62(4):e1-50

27. Reboli AC, Rotstein C, Pappas PG, Chapman SW, Kett DH, Kumar D, Betts R, Wible M, Goldstein BP, Schranz J, Krause DS, Walsh TJ, Anidulafungin SG. Anidulafungin versus fluconazole for invasive candidiasis. N Engl J Med. 2007;356(24):2472-82.

28. Krause DS, Simjee AE, van Rensburg C, Viljoen J, Walsh TJ, Goldstein BP, Wible M, Henkel T. A randomized, double-blind trial of anidulafungin versus fluconazole for the treatment of esophageal candidiasis. Clin Infect Dis. 2004;39(6):770-5.

29. Lewis RE. Current concepts in antifungal pharmacology. Mayo Clin Proc. 2011;86(8):805-17.

\section{Submit your next manuscript to BioMed Central and we will help you at every step:}

- We accept pre-submission inquiries

- Our selector tool helps you to find the most relevant journal

- We provide round the clock customer support

- Convenient online submission

- Thorough peer review

- Inclusion in PubMed and all major indexing services

- Maximum visibility for your research

Submit your manuscript at www.biomedcentral.com/submit
C Biomed Central 